\title{
A note on the effects of epidemic forecasts on epidemic dynamics
}

\author{
Nicholas R Record ${ }^{\text {Corresp., Equal first author, } 1 \text {, Andrew Pershing }}{ }^{\text {Equal first author, } 2}$ \\ ${ }^{1}$ Bigelow Laboratory for Ocean Sciences, East Boothbay, ME, United States of America \\ ${ }^{2}$ Gulf of Maine Research Institute, Portland, ME, USA \\ Corresponding Author: Nicholas R Record \\ Email address: nrecord@bigelow.org
}

The purpose of a forecast, in making an estimate about the future, is to give people information to act on. In the case of a coupled human system, a change in human behavior caused by the forecast can alter the course of events that were the subject of the forecast. In this context, the forecast is an integral part of the coupled human system, with two-way feedback between forecast output and human behavior. However, forecasting programs generally do not examine how the forecast might affect the system in question. This study examines how such a coupled system works using a model of viral infection -- the susceptible-infected-removed (SIR) model -- when the model is used in a forecasting context. Human behavior is modified by making the contact rate responsive to other dynamics, including forecasts, of the SIR system. This modification creates two-way feedback between the forecast and the infection dynamics. Results show that a faster rate of response by a population to system dynamics or forecasts leads to a significant decline in peak infections. Responding to a forecast leads to a lower infection peak than responding to current infection levels. Inaccurate forecasts can lead to either higher or lower peak infections depending on whether the forecast under- or over-estimates the peak. The direction of inaccuracy in a forecast determines whether the outcome is better or worse for the population. While work is still needed to constrain model functional forms, forecast feedback can be an important component of epidemic dynamics that should be considered in response planning. 


\title{
A note on the effects of epidemic forecasts on epidemic dynamics
}

\author{
Nicholas R. Record, Andrew J. Pershing
}

\begin{abstract}
The purpose of a forecast, in making an estimate about the future, is to give people information to act on. In the case of a coupled human system, a change in human behavior caused by the forecast can alter the course of events that were the subject of the forecast. In this context, the forecast is an integral part of the coupled human system, with two-way feedback between forecast output and human behavior. However, forecasting programs generally do not examine how the forecast might affect the system in question. This study examines how such a coupled system works using a model of viral infection - the susceptible-infected-removed (SIR) model - when the model is used in a forecasting context. Human behavior is modified by making the contact rate responsive to other dynamics, including forecasts, of the SIR system. This modification creates two-way feedback between the forecast and the infection dynamics. Results show that a faster rate of response by a population to system dynamics or forecasts leads to a significant decline in peak infections. Responding to a forecast leads to a lower infection peak than responding to current infection levels. Inaccurate forecasts can lead to either higher or lower peak infections depending on whether the forecast under- or over-estimates the peak. The direction of inaccuracy in a forecast determines whether the outcome is better or worse for the population. While work is still needed to constrain model functional forms, forecast feedback can be an important component of epidemic dynamics that should be considered in response planning.
\end{abstract}

\section{Introduction}

The field of forecasting is growing rapidly to include many systems (Payne et al. 2017). This growth has magnified many ethical considerations around forecast development, such as conflicts of interest, representation of uncertainty, equity for end users, and unintended consequences, to name a few (Hobday et al. 2019). One challenge that is becoming more common is that forecasts increasingly deal with systems that have human components. In conventional forecasts, such as weather forecasts, human responses do not measurably affect the system in question (Fig 1A). There are more and more cases, however, where human actions are coupled to the system dynamics. Examples include forecasts used in living resource management (Tommasi et al. 2017), endangered species management (Ross et al. 2020), and epidemic forecasts (Adam 2020). Historically, cases like these have often been framed as projections, which present scenarios that are contingent upon different human choices (Fig 1B).

In contrast to projections, forecasts provide specific predictions about future events. If humans are changing their behavior in response to information in the forecast, and if that change in behavior alters the outcome of the forecast target, then the forecast is no longer outside of the system, but is instead a dynamically connected component of the system (Fig 1C). This phenomenon, sometimes refered to as "forecast feedback" or "reflexive prediction" has been historically considered to some degree in economic and political forecasting (Galatin 1976), but is rarely considered in biological systems. According to the "Law of Forecast Feedback", this type of dynamic can hypothetically produce a recursion paradox, where predictability breaks down entirely (Smith 1964). The complexity of 
41 dealing with forecast feedback or reflexive prediction is a subject of scientific debate, and

42 it's not clear whether a reflexive forecast could ever be verified empirically (Kopec 2011).

43 As forecasts are increasingly used within the systems they are forecasting, we need to

44 examine the effects that forecasts can have on the dynamics of the system in question.

45 Epidemic models represent a case where forecasts and human behavior can be very tightly

46 coupled. A forecast predicting a high infection peak can motivate measures like quarantine

47 or social distancing. These measures change the forecasted dynamics, ideally lowering the

48 predicted peak. A less severe outlook can then motivate an easing of measures, which in

49 turn can raise the peak again. This two-way feedback illustrates the difficulty in forecasting

50 systems coupled to human dynamics.

51 Epidemic models are conventionally used for projections, rather than for forecasts. They

52 can estimate the consequences of certain actions, potential dangers, or scenarios. However,

53 in the modern information-rich environment, there is a demand for real-time forecasts for

54 both management and general audiences, and such forecasts of epidemics are increasingly

55 common. Many of the modeling studies that have responded to the COVID-19 outbreak are

56 forecasts (COVID and Murray 2020; Roosa et al. 2020; Fanelli and Piazza 2020). The United

57 States Center for Disease Control has even issued an open call for COVID-19 forecasts

58 through a forecasting challenge (https://github.com/cdcepi/COVID-19-ILI-forecasting),

59 and many research teams and news sites serve forecasts to the public in real time. This

60 raises the question: what is the effect of a forecast on the epidemic dynamics? In most

61 biological forecasts, this type of feedback is not explicitly taken into account.

62 We examined this question using the standard susceptible-infected-removed (SIR)

63 epidemic model. The SIR framework underlies many projections and forecasts that form

64 the basis for response strategies for COVID-19 and other epidemics (Adam 2020). We built

65 forecast feedback into the SIR model by allowing the contact rate to change dynamically in

66 response to different information provided by the SIR equations. In turn, the SIR model

67 dynamics depend on the contact rate, so that there is two-way feedback between the

68 forecast and the other system dynamics. The purpose here is not to fully analyze or characterize the global properties of a particular dynamical model, as there is a whole class of well characterized versions of the SIR model that consider a wide range of additional processes and details (Beretta and Takeuchi 1995; Anderson and May 1979; Takeuchi, Ma, and Beretta 2000; Satsuma et al. 2004; Kyrychko and Blyuss 2005; Batista 2020) that one could modify to explore these feedbacks. Rather, we use simulations of a few simple SIR modifications to illustrate important implications of these feedbacks. Examining a system in this way provides a critical analysis of the role of a forecast in a rapidly changing coupled human system.

Our objective is to test the potential effects of an epidemic forecast on the epidemic itself.

79 The approach is to explore this question in its simplest form. To that end, we use the

80 standard susceptible-infected-removed (SIR) model: 


$$
\begin{aligned}
& \frac{d S}{d t}=-\alpha S I \\
& \frac{d I}{d t}=\alpha S I-\beta I \\
& \frac{d R}{d t}=\beta I
\end{aligned}
$$

82 Here, $S$ is the number of individuals in a population that are susceptible to infection, $I$ is the

83 number of infected individuals, and $R$ is the number of removed individuals (interpreted as

84 recovered/immune, dead, or otherwise removed); $\alpha$ is the contact rate, and $\beta$ is the

85 removal rate. The SIR model has been one of the primary tools for making simulations on

86 which control and intervention measures are based, such as social distancing, and shelter

87 in place measures, though other statistical and machine learning tools are also used. There

88 are many ways to modify this system of equations to better approximate a particular

89 epidemic. Our interest is in the general effect that model output (either current or

90 forecasted) can have on the dynamics themselves, as mediated through changes in human

91 behavior. The contact rate $\alpha$ is what would likely change with the suggested response measures. A high infection or a severe enough forecast are what motivates measures whose aim is to reduce the contact rate. We therefore test the effect of allowing $\alpha$ to vary as a function of the SIR model output, in a way that is dynamically linked to the other model equations.

Our examination of this system asks two general questions:

(1) What if adoption of response measures depends on the current level of infection? This simulates the scenario where the human response is different depending on whether

(2) What if adoption of response measures depends on the forecasted peak infection? Peak infection is a primary concern with major epidemics because of health care capacity. In this case, $\alpha$ depends on the predicted peak infection $I_{*}$, with the prediction made at any given time using a standard SIR solution. $\alpha$ :

$$
\begin{array}{cc}
\frac{d S}{d t} & =-\alpha S I \\
\frac{d I}{d t} & =\alpha S I-\beta I \\
\frac{d R}{d t} & =\beta I \\
\frac{d \alpha}{d t} & =r \alpha(1-\alpha / K)
\end{array}
$$


108 The fourth equation is formulated so that $\alpha$ approaches an asymptotic contact rate $K$. This

109 is a common functional form in ecological theory (Record, Pershing, and Maps 2014). There 110 are likely multiple possible functional forms for $K$. There is some evidence that the contact 111 rate is responsive to infection dynamics in an approximately monotonic way. For example, 112 when government response indices (Hale et al. 2020) are taken as a proxy for contact rate, 113 and the infection level is scaled to the maximum for each country, a negative relationship 114 appears (Fig 2A). To make the asymptotic contact rate responsive to the infection 115 dynamics, $K$ is a function of $I$ :

$$
K(I)=\frac{\alpha_{0}-\alpha_{*}}{N^{2}} I^{2}+\alpha_{*}
$$

The two parameters $\alpha_{*}$ and $\alpha_{0}$ are respectively the unhindered contact rate, and the

118

119

120

121

122

123

124

125

126

127

128

129

131

132

133 reduced contact rate after response measures are in full effect. This gives a declining curve in asymptotic contact rate $K$ as a function of the infection level $I$ (Fig 2B). Finally, we have the parameter $r$, which determines how quickly people adopt response measures-i.e. how quickly they move from one $\alpha$ to another. It is likely that from an empirical point of view, $r$ could have a wide range of values, depending on the community and other contextual information. There are other possibilities for the shape of this curve and for the $\alpha$ equation, with different functional forms and producing a wide range of possible dynamics. The quadratic gives a simple curve where the response gets more intense as infections get higher, which allows us to simulate some simple but realistic properties. We use the model in normalized form to show general results, rather than those specific to a particular outbreak (Parameter values: $\alpha_{*}=1, \alpha_{0}=0.2, \beta=0.1$; Initial conditions: $S=0.999$, $I=0.001, R=0, \alpha=1$ ). With the equations normalized, the total population size is always $N=1$. As a diagnostic, we use $I_{\text {max }}$, the magnitude of the peak infection as the dynamics play out, because of its relevance to health care capacity, as in the COVID-19 pandemic.

Question (2) requires having a forecast. For this, we use an analytical solution for the infection peak (Weiss 2013) of the SIR model,

$$
I_{*}=\left[1-\frac{\beta}{\alpha N}-\frac{\beta}{\alpha N} \log \frac{\alpha N}{\beta}\right] N
$$

where $I_{*}(t)$ is the predicted maximum $I$ value-i.e. a forecast of the magnitude of the infection peak, where the forecast is made at any time, $t$. Here, we suppose that people are reacting to the forecasted peak, rather than the current level of infection, and that the forecast is based on current information at any given time. The forecast solves the standard SIR model, using the current parameter values, as a forecaster would do in real time. This is modeled by replacing $I$ with $I_{*}$ in the equation for $K$. Again, we use the realized $I_{\max }$ as a diagnostic.

The objective here is to illustrate potential dynamics of the feedback between forecast output and human behavior, rather than to ananlyze the global properties of this particular formulation. To this end, we step through simulations where we adjust the human response rate parameter $r$ and compare the diagnostic $I_{\max }$. Each simulation represents a 
146 different value for $r$. Additionally, the forecasted $I_{*}$ value at any time represents a

147 hypothetical at time $t$, because the population is constantly responding, and so the forecast

148 is changing. Moreover, as with any forecast, there is the potential that the prediction is an

149 over- or under-estimation of what will happen, due to stochasticity and other forms of

150 uncertainty. Forecast error can potentially affect forecast feedback dynamics. We test this

151 effect by scaling the forecasted $I_{*}$ value proportionally, from 0.4 to 1.6 , and looking at the

152 effect on the infection peak $I_{\max }$ across a range of $r$ values. Here, each simulation

153 represents a different accuracy offset.

154

155

156

157

158

159

160

161

162

163

164

165

166

167

168

169

170

171

172

173

174

175

176

177

178

179

180

181

182

183

\section{Results}

We evaluate question (1) by looking at how $I_{\max }$ depends on $r$ (Fig 3A). Responding to the current level of infection leads to a drop in the infection peak (approximately $8 \%$ using this set of parameters), as compared to no response, if the response rate is sufficiently fast. If the response rate is very slow, there is effectively no reduction in the infection peak. There is a sharp transition between these two cases. In this example, a response rate slower than about $r=0.1$ - corresponding to a response time of about 10 time units - makes no notable change in the infection peak. For different parameter values, the threshold levels can be different, but the basic shape of these relationships is consistent across different parameterizations.

When the contact rate is responsive to the forecasted infection peak rather than to the instantaneous infection level (question 2), the shape of the curve is similar (Fig 3A). The reduction in the peak infection is larger, (approximately $\sim 12 \%$ drop using this set of parameters, or roughly a $50 \%$ improvement over the former case). There is also a notable difference in the needed response time. In this case, a response time of 10 time units ( $r=0.1$ ) is sufficient for most of the drop to occur. Across parameter space, the magnitudes of these differences change, but the pattern is consistent.

We tested the effects of an over- or under-estimate forecast (Fig 3B), with the forecasted value $I_{*}$ scaled proportionally in the range 0.4 to 1.6 . If the forecast is an overestimate, and if the response rate is fast enough, an inaccurate forecast can lower the peak even more than an accurate forecast. An overestimated forecast also gives a larger window of time for response - i.e. the response rate does not need to be as fast to see a lowering of the peak. If the forecast is an underestimate, however, it leads to a higher peak and narrows the response time window. Finally, if the response rate is very slow, inaccuracies in the forecast in either direction make very little difference. In all of these cases, responding to a forecast is better than not responding at all, due to how we have formulated $K(I)$ as a decreasing function of $I$.

\section{Discussion}

Many new forecasting products are coming online. With the COVID-19 pandemic in particular, studies and online tools with real-time or near-real-time preditions and

Peer] reviewing PDF | (2020:04:48416:2:0:NEW 9 Jul 2020) 
184 projections are produced and available rapidly (Biswas and Sen 2020; Batista 2020; Chen, 185 Lu, and Chang 2020; Simha, Prasad, and Narayana 2020; Teles 2020). People are generally 186 not accustomed to interpreting forecasts as being dynamically linked with their own 187 behavioural responses to the forecast, but such cases are more and more common, and 188 include epidemics, climate change, and endangered species management, to name a few.

189 One growing challenge is the blurring lines between forecasts and projections. For COVID19 , early warnings of a potential global pandemic did not motivate early responses in many places, allowing the virus to move from a local to a global problem. In places like the U.S. and U.K., significant action at the national level did not take place until after Imperial College released projections that millions would die in the absence of social distancing (Adam 2020; Dyer 2020), and after signs of the epidemic were widespread. Once actions were taken, the true trajectory began to deviate from the worst case scenarios, and the projections no longer matched reality. This is typical of projections, which aren't intended to be specific predictions, but rather play out different scenarios that depend on initial actions. Climate projections are similar, calculating future conditions based on

200

201

202

203 Representative Concentration Pathways (RCPs) (Taylor, Stouffer, and Meehl 2012). The RCPs each depict a scenario of future carbon such as aggressive emission reductions (RCP2.6) or business as usual (RCP8.5). There is no weighting placed on which pathway society is likely to select. The goal of the projections is to evaluate the consequences of actions and to contrast action with inaction.

Forecasts, on the other hand, do represent specific predictions. We are more accustomed to weather forecasts, where human responses do not change the outcome. Many epidemic models are now offering forecasts (e.g. COVID and Murray 2020; Roosa et al. 2020; Fanelli and Piazza 2020), and in systems like these, humans are a dynamic component, responding to ongoing changes. The human actions depend on the state of the system and the information in forecasts. In reality, not all scenarios are equally available - the likelihood that humans will act depends on the state of the system and the information in forecasts. Examining this dynamic within an SIR model framework illustrates some significant points.

First, as might be expected, using information from a forecast can reduce the magnitude of the infection peak. Additionally, using the forecast also grows the window of time in which a response has an effect. This could give more room to allow for missteps during times when uncertainty is high. Second, inaccuracies in the forecast have an asymmetrical effect on the outcome. An overestimate in the forecast can improve the outcome, in this case lowering the infection peak, but an underestimate in the forecast can actually make things worse. By underestimating the potential danger, response is diminished. Similarly, if the response rate is too slow, a forecast makes very little difference, but an over-estimate forecast can buy more response time. These asymmetries raise the difficult ethical question of whether it is better to give an accurate forecast if a more dire forecast could actually motivate a stronger response. This question becomes even more complex if the forecast is one member in an ensemble that is used for decision making, or is one member in a sequence of forecasts where trust in the forecast is cumulative. If the goal of a forecast is to alter human behavior, then a self-defeating forecast might actually be desirable (Sabetta 2019). In this broader view, accuracy should be considered only one part of a forecast's performance. 


\section{Conclusion}

229 We have to be cautious of using forecasts that do not take into account the effects of

230 forecast feedback on the system dynamics. This is especially important in systems where

231 human health depends on the forecast, as is the case with SIR and other epidemic models.

232 The examples shown here represent just one possible formulation for this type of dynamic.

233 Future work is required to better constrain the possible functional forms, both empirically

234 and theoretically. There is a whole class of models with forecast feedback modifications to

235 examine - nearly any dynamical model where human decision making potentially plays an

236 integrated role - producing a wide range of model behaviors. Forecast feedback creates

237 certain challenges and limitations for predictions, possibly paradoxical, but at a minimum

238 forecasters should try to understand the effects this feedback can have on the systems we

239 are trying to predict.

240

241

242

243

\section{References}

245

246

247

248

249

250

251

252

253

254

255

256

257

258

259

260 19." Nature. medRxiv.

\section{Acknowledgements}

We would like to acknowledge the institutions around the world that are working hard to make epidemic data and forecasts freely available. We thank the editor and two reviewers for helpful comments.

Adam, D. 2020. "Special Report: The Simulations Driving the World's Response to Covid-

Anderson, Roy M, and Robert M May. 1979. "Population Biology of Infectious Diseases: Part I." Nature 280 (5721). Nature Publishing Group: 361-67.

Batista, Milan. 2020. "Estimation of the Final Size of the Covid-19 Epidemic." Preprint.

Beretta, Edoardo, and Yasuhiro Takeuchi. 1995. "Global Stability of an Sir Epidemic Model with Time Delays." Journal of Mathematical Biology 33 (3). Springer: 250-60.

Biswas, Kathakali, and Parongama Sen. 2020. "Space-Time Dependence of Corona Virus (Covid-19) Outbreak." arXiv Preprint arXiv:2003.03149.

Chen, Yi-Cheng, Ping-En Lu, and Cheng-Shang Chang. 2020. "A Time-Dependent Sir Model for Covid-19." arXiv Preprint arXiv:2003.00122.

COVID, IHME, and Christopher JL Murray. 2020. "Forecasting Covid-19 Impact on Hospital Bed-Days, Icu-Days, Ventilator-Days and Deaths by Us State in the Next 4 Months." medRxiv. Cold Spring Harbor Laboratory Press. 
261 Dyer, Owen. 2020. "Covid-19: US Testing Ramps up as Early Response Draws Harsh

262 Criticism." British Medical Journal Publishing Group.

263 Fanelli, Duccio, and Francesco Piazza. 2020. "Analysis and Forecast of Covid-19 Spreading

264 in China, Italy and France." Chaos, Solitons \& Fractals 134. Elsevier: 109761.

265 Galatin, Malcolm. 1976. "Optimal Forecasting in Models with Uncertainty When the

266 Outcome Is Influenced by the Forecast.” The Economic Journal 86 (342). JSTOR: 278-95.

267 Hale, Thomas, Anna Petherick, Toby Phillips, and Samuel Webster. 2020. "Variation in

268 Government Responses to Covid-19." Blavatnik School of Government Working Paper 31.

269 Hobday, Alistair J, Jason R Hartog, John P Manderson, Katherine E Mills, Matthew J Oliver,

270 Andrew J Pershing, and Samantha Siedlecki. 2019. "Ethical Considerations and

271 Unanticipated Consequences Associated with Ecological Forecasting for Marine Resources."

272 ICES Journal of Marine Science 76 (5). Oxford University Press: 1244-56.

273 Kopec, Matthew. 2011. "A More Fulfilling (and Frustrating) Take on Reflexive Predictions."

274 Philosophy of Science 78 (5). University of Chicago Press Chicago, IL: 1249-59.

275 Kyrychko, Yuliya N, and Konstantin B Blyuss. 2005. "Global Properties of a Delayed Sir

276 Model with Temporary Immunity and Nonlinear Incidence Rate." Nonlinear Analysis: Real

277 World Applications 6 (3). Elsevier: 495-507.

278 Payne, Mark R, Alistair J Hobday, Brian R MacKenzie, Desiree Tommasi, Danielle P

279 Dempsey, Sascha MM Fässler, Alan C Haynie, Rubao Ji, Gang Liu, Patrick D Lynch, Daniela

280 Matei, Anna K Miesner, Katherine E Mills, Kjersti O Strand and Ernesto Villarino. 2017.

281 "Lessons from the First Generation of Marine Ecological Forecast Products." Frontiers in

282 Marine Science 4. Frontiers: 289.

283 Record, Nicholas R, Andrew J Pershing, and Frédéric Maps. 2014. "The Paradox of the

284 'Paradox of the Plankton'." ICES Journal of Marine Science 71 (2). Oxford University Press:

285 236-40.

286 Roosa, K, Y Lee, R Luo, A Kirpich, R Rothenberg, JM Hyman, P Yan, and G Chowell. 2020.

287 "Real-Time Forecasts of the Covid-19 Epidemic in China from February 5th to February

288 24th, 2020." Infectious Disease Modelling 5. Elsevier: 256-63.

289 Ross, Camille, Nicholas R Record, Benjamin Tupper, David Brickman, and Daniel E

290 Pendleton. 2020. "Predicting Regions of North Atlantic Right Whale, Eubalaena Glacialis,

291 Habitat Suitability in the Gulf of Maine in 2050." Elementa: Journal of the Anthropocene in

292 review.

293 Sabetta, Lorenzo. 2019. "Self-Defeating Prophecies: When Sociology Really Matters." In

294 Anticipation, Agency and Complexity, 51-59. Springer.

295 Satsuma, Junkichi, R Willox, A Ramani, B Grammaticos, and AS Carstea. 2004. "Extending

296 the Sir Epidemic Model." Physica A: Statistical Mechanics and Its Applications 336 (3-4).

297 Elsevier: 369-75. 
298 Simha, Ashutosh, R Venkatesha Prasad, and Sujay Narayana. 2020. "A Simple Stochastic Sir 299 Model for Covid 19 Infection Dynamics for Karnataka: Learning from Europe." arXiv 300 Preprint arXiv:2003.11920.

301 Smith, George Cline. 1964. "The Law of Forecast Feedback." The American Statistician 18 302 (5). Taylor \& Francis Group: 11-14.

303 Takeuchi, Yasuhiro, Wanbiao Ma, and Edoardo Beretta. 2000. "Global Asymptotic

304 Properties of a Delay Sir Epidemic Model with Finite Incubation Times." Nonlinear Analysis:

305 Theory, Methods \& Applications 42 (6). Pergamon: 931-47.

306 Taylor, Karl E, Ronald J Stouffer, and Gerald A Meehl. 2012. "An Overview of Cmip5 and the 307 Experiment Design." Bulletin of the American Meteorological Society 93 (4). American 308 Meteorological Society: 485-98.

309 Teles, Pedro. 2020. "Predicting the Evolution of Covid-19 in Portugal Using an Adapted Sir 310 Model Previously Used in South Korea for the Mers Outbreak." medRxiv. Cold Spring Harbor 311 Laboratory Press.

312 Tommasi, Desiree, Charles A Stock, Alistair J Hobday, Rick Methot, Isaac C Kaplan, J Paige 313 Eveson, Kirstin Holsman, Timothy J. Miller, Sarah Gaichas, Marion Gehlen, Andrew 314 Pershing,Gabriel A Vecchi, Rym Msadek, Tom Delworth, C Mark Eakin, Melissa A Haltuch, 315 Roland Séférian,Claire M Spillman, Jason R Hartog, Samantha Siedlecki, Jameal F Samhouri, 316 Barbara Muhling,Rebecca G Asch, Malin L Pinsky, Vincent S Saba, Sarah B Kapnick, Carlos F 317 Gaitan,Ryan R Rykaczewski, Michael A Alexander, Yan Xue, Kathleen V Pegion, Patrick 318 Lynch,Mark R Payne, Trond Kristiansen, Patrick Lehodey, Francisco E Werner. 2017. 319 "Managing Living Marine Resources in a Dynamic Environment: The Role of Seasonal to 320 Decadal Climate Forecasts." Progress in Oceanography 152. Elsevier: 15-49.

321 Weiss, Howard Howie. 2013. "The Sir Model and the Foundations of Public Health." 322 Materials Mathematics, 0001-0017. 
324 Figure 1. (A) The conventional forecasting scheme, where a system informs a forecast, which 325 informs some human response. (B) The conventional scheme for projection, where a range of 326 possible actions produce multiple scenarios (i.e. projections), which can inform action. (C) The 327 forecasting scheme where the human response is part of the system dynamics - i.e. forecast 328 feedback or reflexive prediction.

329 Figure 2. (A) Evidence of a relationship between the magnitude of infection and a contact rate 330 index. The contact rate is the inverse of the government response index from Hale et al. 331 (2020), so that a higher index corresponds to a higher contact rate. Infection magnitude is scaled to the maximum for each country. (B) The dependence of the asymptotic contact rate (K) on the population size (I).

Figure 3. (A) The dependence of the infection peak magnitude on the rate (r) at which the population reduces the contact rate (i.e. adopts response measures). The population responding to current infection levels (black); the population responding to forecasted infection peaks (grey). (B) The dependence of the infection peak levels (contours) on the rate (r) at which the population reduces the contact rate (e.g. adopts social distancing behaviors) and the accuracy of the forecast. In this simulation, the population is responding to forecasted 340 peak infection levels. 


\section{Figure 1}

Forecasting schematic diagram

(A) The conventional forecasting scheme, where a system informs a forecast, which informs some human response. (B) The conventional scheme for projection, where a range of possible actions produce multiple scenarios (i.e. projections), which can inform action. (C) The forecasting scheme where the human response is part of the system dynamics -- i.e. forecast feedback or reflexive prediction.

\section{A}

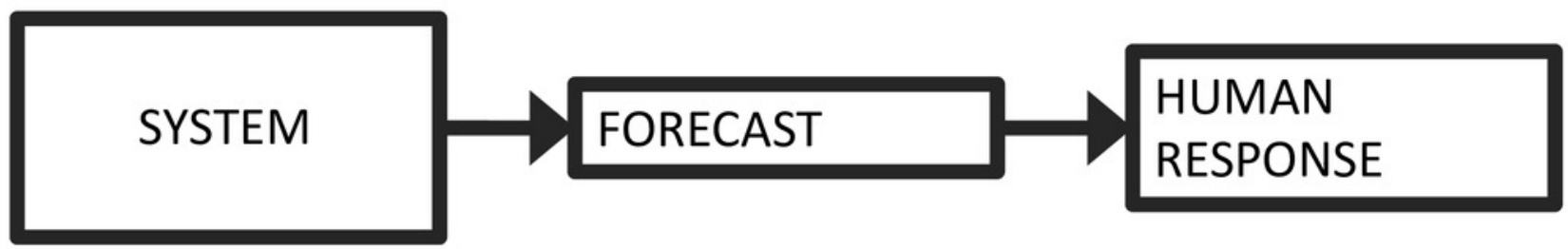

B

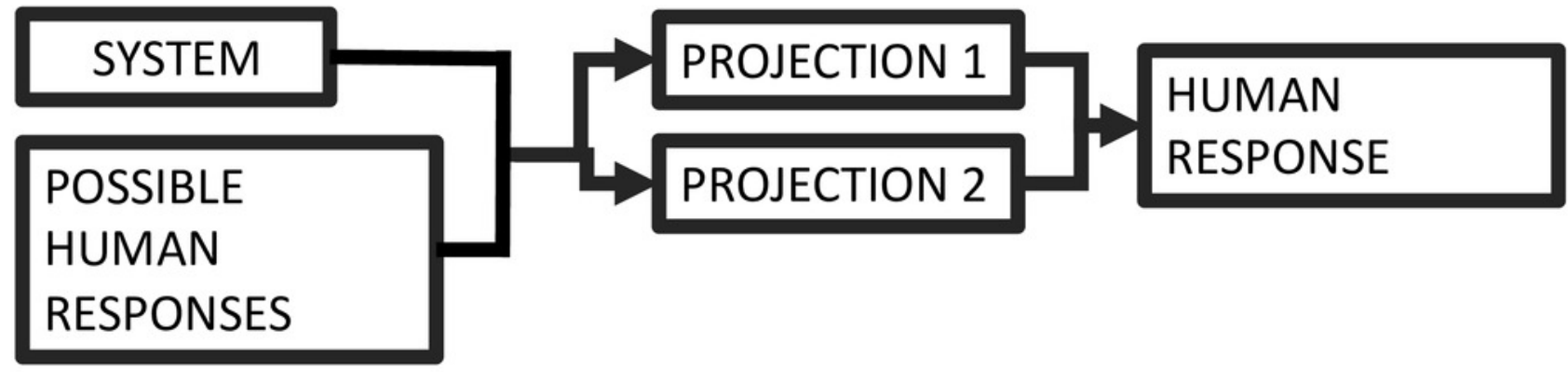

C

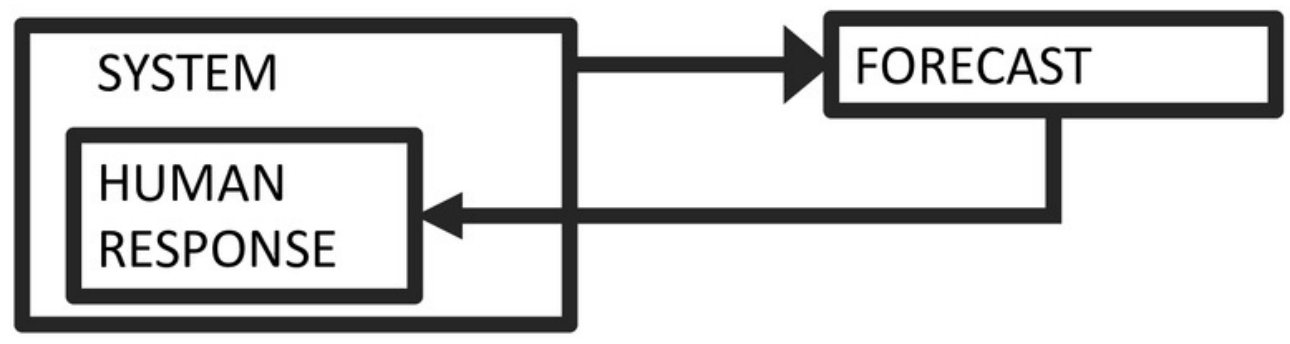


Figure 2

Plots informing the model structure.

(A) Evidence of a relationship between the magnitude of infection and a contact rate index.

The contact rate is the inverse of the government response index from Hale et al. (2020), so that a higher index corresponds to a higher contact rate. Infection magnitude is scaled to the maximum for each country. (B) The dependence of the asymptotic contact rate (K) on the population size (I).

A

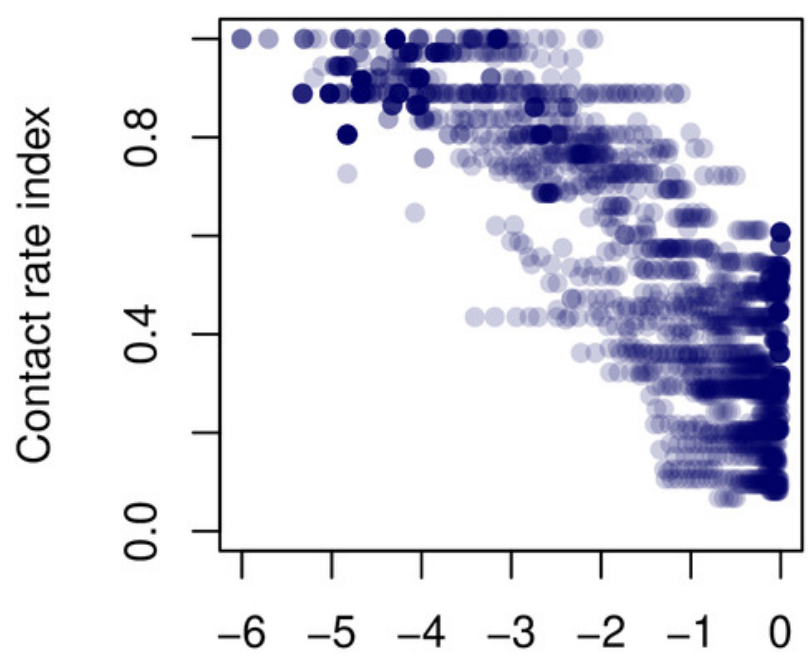

Cases (log proportion of max)
B

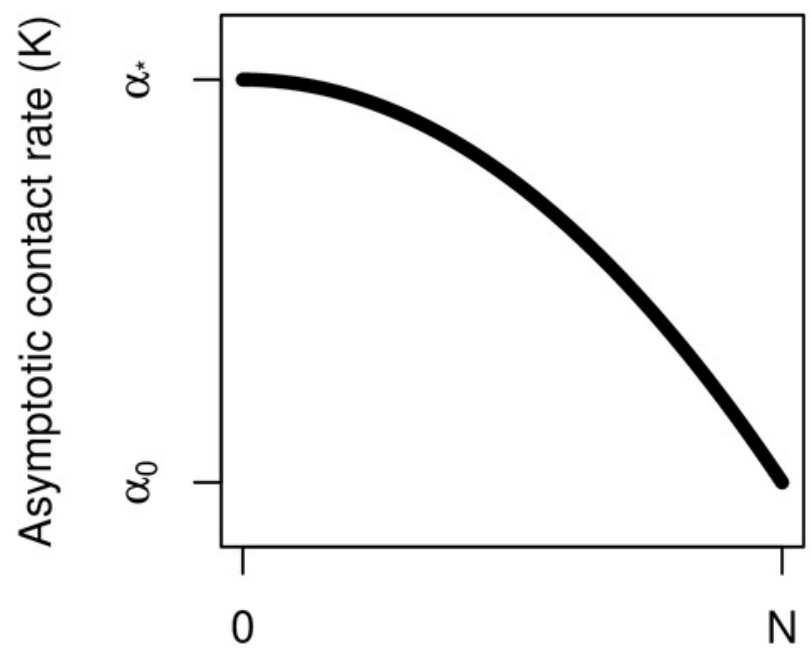

Infected population (I) 
Figure 3

Results of model analysis.

(A) The dependence of the infection peak magnitude on the rate ( $r$ ) at which the population reduces the contact rate (i.e. adopts response measures). The population responding to current infection levels (black); the population responding to forecasted infection peaks (grey). (B) The dependence of the infection peak levels (contours) on the rate ( $r$ ) at which the population reduces the contact rate (e.g. adopts social distancing behaviors) and the accuracy of the forecast. In this simulation, the population is responding to forecasted peak infection levels.

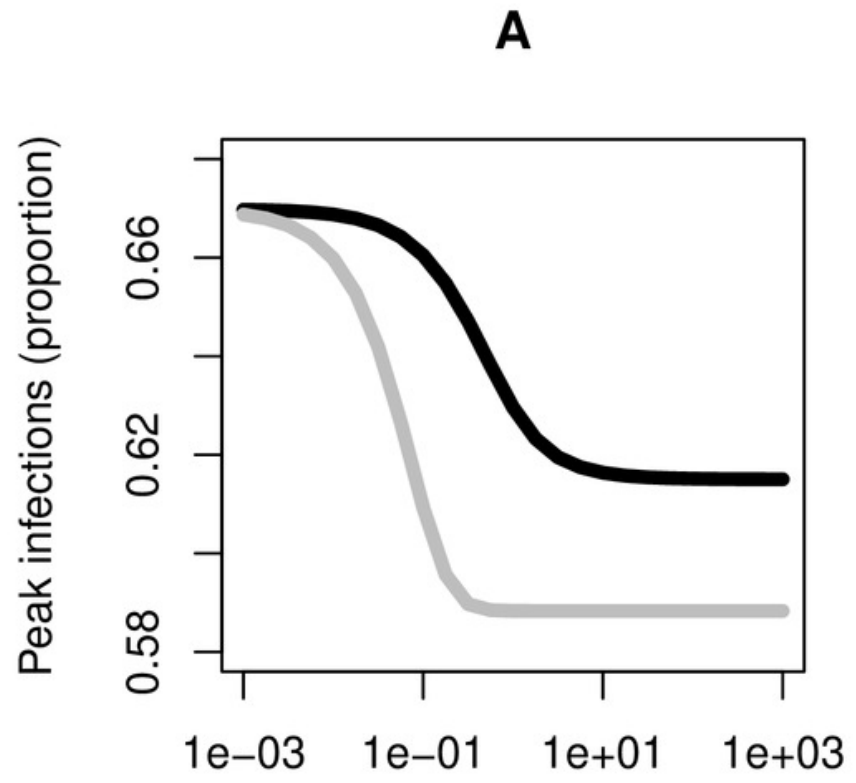

Response rate ( $r)$ (1/unit time)

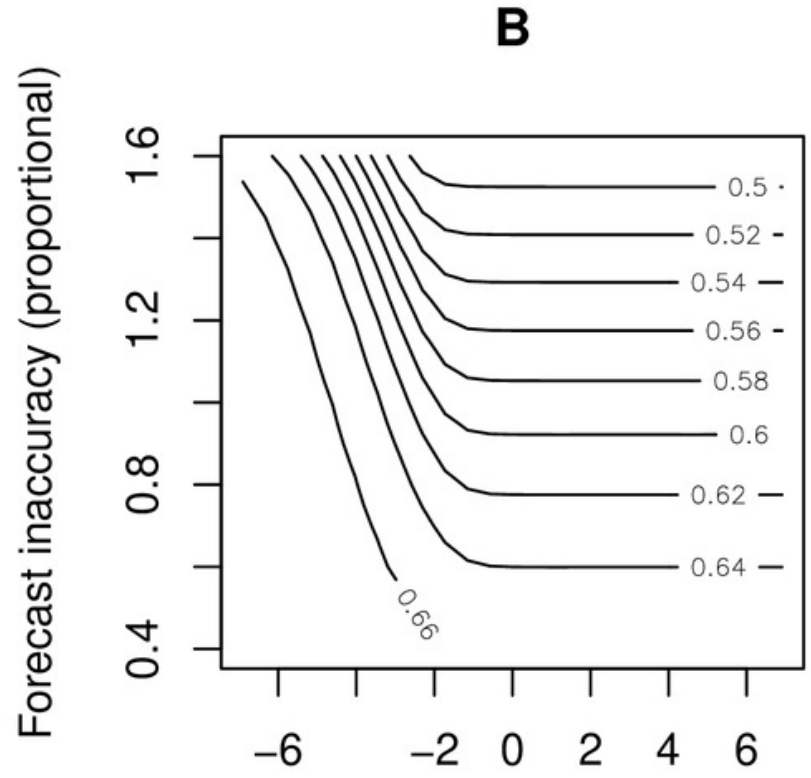

Response rate (log 1/unit time) 